\title{
Scaling for faster macroparticle simulation in longitudinal multiparticle dynamics
}

\author{
J. A. MacLachlan and Z. Nazario* \\ Fermi National Accelerator Laboratory, Box 500, Batavia, Illinois 60510
}

(Received 27 July 2000; published 3 November 2000)

\begin{abstract}
Macroparticle tracking is a direct and attractive approach to following the evolution of a longitudinal phase space distribution. When the particles interact through short-range wake fields or when the interparticle force is included, calculations of this kind require a large number of macroparticles. However, it is possible to reduce both the number of macroparticles required and the number of tracking steps per unit simulated time by employing a simple scaling. It is demonstrated that the Vlasov equation is unchanged by introduction of the scaled quantities. It is further shown that under rather general conditions the speed of calculation improves with the fourth power of the scaling constant. Two examples comparing scaled and original cases illustrate the effectiveness of the approach. Limitations to the amount of rescaling are discussed.
\end{abstract}

PACS numbers: 29.27.Bd, 02.60.Cb

\section{INTRODUCTION}

Multiparticle tracking programs have a long history and established utility for modeling the evolution of the longitudinal phase space distributions for particles in accelerators as the particles respond to the $\mathrm{rf}$ in acceleration or bunch manipulation. The macroparticle distribution can be used to approximate the evolution of the beam current distribution or Fourier spectrum throughout the process being modeled. Passing from single particle dynamics to multiparticle dynamics by calculating the beam current every time step and including the contribution of the fields induced by it to the single particle motion is a direct extension of the technique which makes a wide range of interesting problems accessible. However, the question of the number of macroparticles needed or the relevant bandwidth for quantities calculated in frequency domain requires careful attention, and it is very easy to generate spectacular spurious instabilities by excessive time steps or an insufficient number of macroparticles. This issue is the source and focus of what follows; recent studies of high brightness injectors and the so-called factory accelerators have given it currency. It will be shown that modeling which might appear to require order $10^{6}$ macroparticles and days of time on a fast computer can be scaled to the desktop and normal patience.

\section{SCALING CONCEPT}

The objective of the scaling sought is to allow more rapid calculation of the time evolution of the energy and rf phase of particles in a synchrotron or storage ring taking into account the contribution of beam image current, direct interparticle force, etc. In a multiparticle tracking calculation, the positions of representative macroparticles

\footnotetext{
*Permanent address: Dept. of Physics, University of Puerto Rico, Rio Piedras Campus, San Juan, Puerto Rico 00931-3343.
}

in an energy-phase plane are found by iterating a pair of first-order difference equations with an iteration step almost always taken as one period of circulation of particles around the ring. The iteration step can be fractional or multiple turns, however. The interaction of the particles is calculated each iteration by an energy increment for each particle arising from the fields generated by the total beam current.

A useful approximation for the single turn map is [1]

$$
\begin{gathered}
\varphi_{i, m}=\varphi_{i, m-1}+\frac{2 \pi h \eta}{\beta_{s}^{2} E_{s}} \varepsilon_{i, m-1}, \\
\varepsilon_{i, m}=\varepsilon_{i, m-1}+e V\left(\varphi_{i, m}+\phi_{s, m}\right)-e V\left(\phi_{s, m}\right),
\end{gathered}
$$

where $\varphi$ is a phase difference between a particle and the synchronous phase $\phi_{s}, \varepsilon$ is the energy difference between a particle and the synchronous energy $E_{s}, i$ labels particles, and $m$ labels turns. All symbols are defined in Table I.

By inspection of the map parameters it is plausible that the phase space motion can be accelerated by scaling up the phase slip factor $\eta$ and the potential by the same factor. Notice that the potential is not necessarily just that from the rf system; the collective potential produced by the beam current enters identically. A trial comparing the evolution of some distribution mapped according to Eqs. (1) and (2) with that obtained from the map with $\eta$ and $V$ multiplied by a scaling factor $\lambda$ reveals that the distributions, i.e., plots of macroparticle locations on the energy-phase plane, are identical when the plot for time $t$ in the unscaled calculation is compared to time $t / \lambda$ in the scaled calculation.

The obvious gain is a factor $\lambda^{-1}$ in the computing time by speeding up the clock in the scaled calculation. However, the scaling up of the time means that frequencies associated with the motion, such as the rf frequency, for example, have also been scaled up. A consequence of the frequency scaling is that a resonant potential in the problem, such as a higher order cavity mode (HOM), for example, must be entered into the calculation with its resonant frequency scaled up by the same factor. If this is done, 
TABLE I. Definition of frequently used symbols.

\begin{tabular}{cl}
\hline \hline Symbol & \\
\hline$\phi$ & rf phase \\
$\phi_{s}$ & Synchronous phase \\
$\varphi_{i}$ & Difference between particle phase and $\phi_{s}$ \\
$i, j$, or $k$ & Index for particles \\
$m$ & Index for turns \\
$h$ & rf harmonic number \\
$e$ & Elementary particle charge $(>0)$ \\
$f$ & Frequency \\
$E_{s}$ & Synchronous energy \\
$\beta$ & Relativistic velocity $v / c$ \\
$\gamma$ & Relativistic energy $E_{s} / m_{0} c^{2}$ \\
$\gamma_{T}$ & $\gamma$ of transition energy in synchrotron \\
$\eta$ & Phase slip factor $\left(\gamma_{T}^{-2}-\gamma^{-2}\right)$ \\
$V$ & Total potential \\
$V_{\mathrm{rf}}$ & Peak rf voltage \\
$V_{\text {coll }}$ & Collective potential \\
$\lambda$ & Dimensionless real scaling constant \\
$Z$ & Longitudinal impedance \\
$f_{0}$ & Beam circulation frequency \\
$\omega_{0}$ & Angular frequency of beam circulation \\
$\omega_{s}$ & Angular frequency of small amplitude synchrotron oscillations \\
$Q_{s}$ & Synchrotron tune \\
$\varepsilon_{j}$ & Difference between energy of $j$ th particle and $E_{s}$ \\
$\tau_{j}$ & Time difference $\varphi_{j} / h \omega_{0}$ \\
$p$ & Index for Fourier harmonics \\
$\hat{\tau}_{k}$ & Amplitude of time excursion in synchrotron oscillation \\
$\psi_{k}$ & Synchrotron oscillation phase of a particle \\
$N$ & Number of particles in beam \\
$\Psi$ & Particle density in phase space \\
\hline \hline & \\
\hline
\end{tabular}

the result of scaled and original mappings may be indistinguishable from one another for a reasonable choice of $\lambda$.

Figures 1 and 2 compare the collective potential versus rf phase for an $h=1$ bunch with $4 \times 10^{13}$ protons in a calculation scaled by $\lambda=2$ with an unscaled modeling. The storage ring parameters used in this and a later example are given in Table II. The parameters are similar to those of the Los Alamos Proton Storage Ring with the addition of a resonance $1 \%$ above the third harmonic of the beam circulation frequency $f_{0}$; it is the only source of collective potential included in this example. Figure 1 is the HOM voltage after $4 \times 10^{4}$ turns $(0.014 \mathrm{~s})$ of tracking; the curve with a dash-dotted representation is the longitudinal charge distribution with the same number of bins used for the potential. Figure 2 shows the same quantities for the scaled model after $2 \times 10^{4}$ turns. Many other features of the distribution can be compared including the time evolution of moments and the phase plane plots, which appear nearly identical in an overlay comparison. Note the factor of 2 scale difference on the potential axis in Figs. 1 and 2 . The scaled charge distribution looks slightly smoother because of faster mixing between adjacent bins in the scaled case.

When broadband impedance or the direct interparticle forces (variously called space charge or perfectly conduct- ing wall forces) are included, the consequences of the frequency scaling are different and very advantageous. If it has been concluded that the effects of beam pipe impedance must be covered over some particular frequency bandwidth, or, more or less equivalently, wake fields down to some minimum range, it will be necessary to bin the charge distribution into bins sufficiently narrow to reflect detail in the distribution to that scale. It is easiest to formulate the requirement in the frequency domain: the bandwidth of interest must be spanned by Fourier components of the beam current. If beam circulation frequency is $f_{0}$ and the required bandwidth is $W$, the charge distribution must be divided into $2 W / f_{0}$ bins for the finite transform. However, in the scaled system $f_{0}$ is $\lambda$ times higher; the number of bins can be reduced by a factor of $\lambda^{-1}$. The number of harmonics is reduced, but the frequency range covered is the same. What has been sacrificed is sensitivity to features in the frequency dependence of the longitudinal impedance $Z_{\|}$on the scale of $f_{0}$. For example, the space charge term in frequency domain is

$$
\frac{Z_{\mathrm{sc}}}{n}=-i \frac{Z_{0} g}{2 \beta \gamma^{2}},
$$

where $n$ is the harmonic number, $Z_{0}=\sqrt{\mu_{0} / \epsilon_{0}}=$ $377 \Omega, \gamma=E / m_{0} c^{2}$, and $g$ is the geometric factor for 




FIG. 1. Voltage vs rf phase (histogram) produced by an $h=3$ HOM resonance in the ring whose parameters are given in Table II, after $4 \times 10^{4}$ turns $(0.014 \mathrm{~s})$. The beam intensity is $4 \times 10^{13}$ protons. The longitudinal charge distribution is shown with the same binning by the dash-dotted curve.

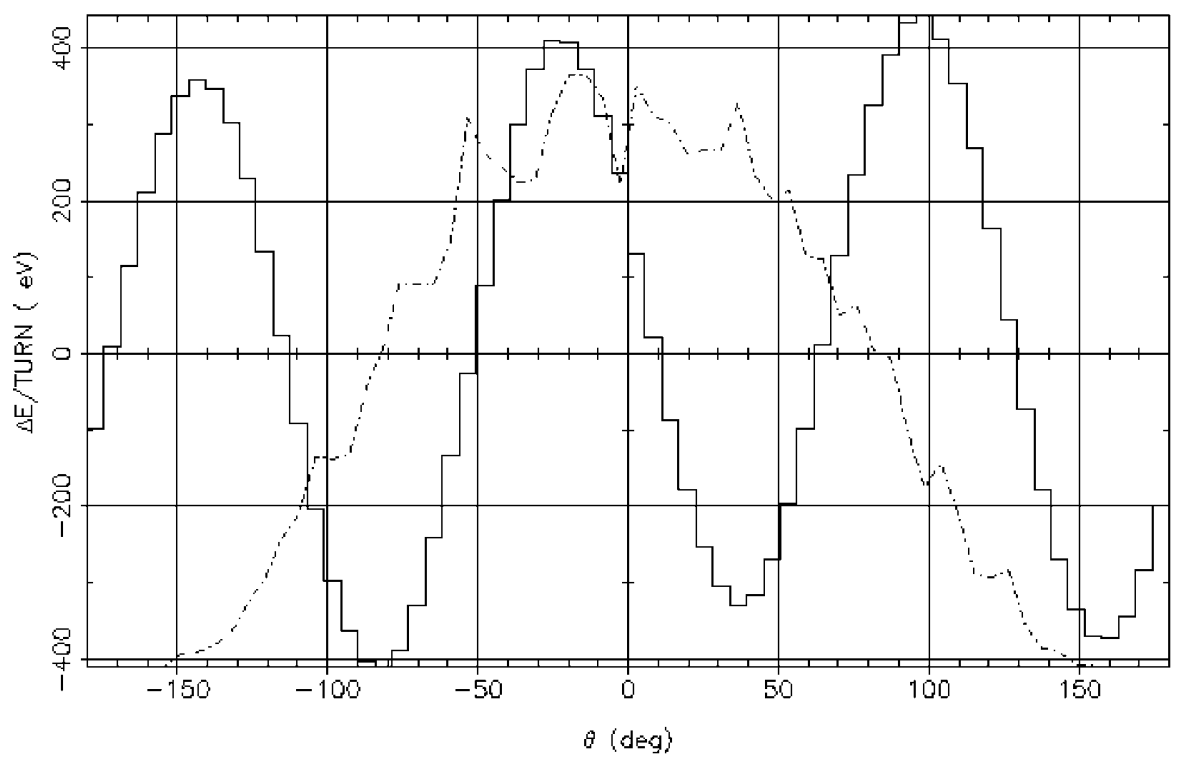

FIG. 2. The conditions are the same as in Fig. 1 scaled with $\lambda=2$ after $2 \times 10^{4}$ iterations The voltage scale is $V^{\prime}=\lambda V$.

the beam tube. The evaluation of this term is not compromised by more widely spaced frequency sampling. For wideband impedances in general, there will be little loss in information for reasonable choices of $\lambda$.

What has been gained is the opportunity to reduce the number of macroparticles required for a given level of numerical noise in the mapping. It has been shown that when a three point difference formula for the derivative of the linear charge density is used in calculating the space charge force in time domain, the number of macroparticles can be reduced by a factor of $\lambda^{-3}$ when the number of bins is reduced by the factor $\lambda^{-1}$ [2]. An argument based on sampling in the frequency domain shows that more generally this result applies to any source of longitudinal impedance with a frequency dependence featureless on the scale of $\Delta f \sim$ few $\times f_{0}$ [3]. Thus the net effect of scaling by a factor of $\lambda$ can be an improvement in the speed of the calculation on the order of $\lambda^{4}$ for most applications. 
TABLE II. Storage ring parameters used in tracking examples.

\begin{tabular}{lccc}
\hline \hline \multicolumn{1}{c}{ Parameter } & Symbol & Value & Units \\
\hline Circumference & $C$ & 90.261 & $\mathrm{~m}$ \\
Momentum & $p$ & 1459.7 & $\mathrm{MeV} / c$ \\
Transition energy $/ m_{0} c^{2}$ & $\gamma_{T}$ & 3.08 & \\
Slip factor $\gamma_{T}^{-2}-\gamma^{-2}$ & $\eta$ & 0.18694 & \\
rf peak voltage & $V_{\mathrm{rf}}$ & 5 & $\mathrm{kV}$ \\
rf harmonic & $h$ & 1 & \\
Beam circulation frequency & $f_{0}$ & 2.7940 & $\mathrm{MHz}$ \\
HOM frequency & $f_{\text {res }}$ & 8.4658 & $\mathrm{MHz}$ \\
HOM impedance at resonance & $R_{\mathrm{sh}}$ & 300 & $\mathrm{Ohm}$ \\
HOM $Q$ & $Q$ & 100 & \\
\hline \hline
\end{tabular}

\section{FORMAL ANALYSIS}

The discussion based on ad hoc scaling of the mapping was not offered as proof that the scaling is valid, rather that it is plausible. Here it is demonstrated that the Vlasov equation for the original problem is unchanged by introducing the scaled variables and parameters. Symbols used in the following are defined in Table I.

Consider a bunch for which the small amplitude synchrotron oscillation frequency $\omega_{s}=\omega_{0} Q_{s}$. The steady state collective voltage experienced by a particle with phase $\phi$ is

$$
\begin{gathered}
V_{\mathrm{coll}}(\phi)=\frac{e \omega_{0}}{2 \pi} \sum_{k=1}^{N} \sum_{p=-\infty}^{\infty} e^{i p \phi / h}\left\{Z\left(p \omega_{0}\right)-\frac{i \omega_{0} \hat{\tau}_{k}}{2}\left[\left(p+Q_{s}\right) Z\left(p \omega_{0}+\omega_{s}\right) e^{i\left(\psi_{k}+Q_{s} \phi / h\right)}\right.\right. \\
\left.\left.+\left(p-Q_{s}\right) Z\left(p \omega_{0}-\omega_{s}\right) e^{-i\left(\psi_{k}+Q_{s} \phi / h\right)}\right]\right\},
\end{gathered}
$$

where the sum over $k$ is taken over all particles. The single particle equations of motion in canonical energytime variables are

$$
\begin{gathered}
\frac{d \varepsilon_{j}}{d t}=\frac{h e \omega_{0}^{2}}{2 \pi}\left[V_{\mathrm{rf}} \cos \phi_{s}+V_{\mathrm{coll}}^{\prime}\left(\phi_{s}\right)\right] \tau_{j}, \\
\frac{d \tau_{j}}{d t}=-\frac{\eta}{\beta^{2} E_{0}} \varepsilon_{j} .
\end{gathered}
$$

The rf potential is sinusoidal with amplitude $V_{\mathrm{rf}}$. The energy increment $e V_{\mathrm{rf}} \sin \phi_{s}$ keeps a particle with phase $\phi_{s}$ synchronous as the guide field is changed or energy is lost to the real part of the longitudinal impedance. The total Hamiltonian for the motion of all of the particles is

$$
\begin{aligned}
H=-\sum_{k=1}^{N}\{ & \frac{\eta}{2 \beta^{2} E_{0}} \varepsilon_{k}^{2} \\
& \left.+\frac{e h \omega_{0}^{2}}{4 \pi}\left[V_{\mathrm{rf}} \cos \phi_{s}+V_{\mathrm{coll}}^{\prime}\left(\phi_{s}\right)\right] \tau_{k}^{2}\right\} .
\end{aligned}
$$

The time evolution of the phase space distribution for the multiparticle system is described by the Vlasov equation

$$
\frac{d \Psi}{d t}=\frac{\partial \Psi}{\partial t}+\frac{\partial \Psi}{\partial \tau} \frac{d \tau}{d t}+\frac{\partial \Psi}{\partial \varepsilon} \frac{d \varepsilon}{d t}=0
$$

where $\Psi(\varepsilon, \tau ; t)$ is the particle distribution function which depends on $t$ only implicitly through $\varepsilon$ and $\tau$. It expresses Liouville's theorem on conservation of phase space density and is valid in the absence of diffusion or external damping of the motion.

The scaling introduced into the map in Sec. II creates an apparently new physical system which will be denoted as the primed system; it is related to the original system by

$$
\eta^{\prime}=\lambda \eta, \quad V^{\prime}=\lambda V, \quad t^{\prime}=t / \lambda
$$

However, if the two systems are physically equivalent, then $\left.\Psi^{\prime}\right|_{\lambda t^{\prime}}=\left.\Psi\right|_{t}$. By direct substitution one finds

$$
H^{\prime}=\lambda H \text {. }
$$

The Vlasov equation in the primed system is

$$
\frac{\partial \Psi^{\prime}}{\partial t^{\prime}}+\frac{\partial \Psi^{\prime}}{\partial \tau^{\prime}} \frac{d \tau^{\prime}}{d t^{\prime}}+\frac{\partial \Psi^{\prime}}{\partial \varepsilon^{\prime}} \frac{d \varepsilon^{\prime}}{d t^{\prime}}=0
$$

which leads immediately to

$$
\lambda \frac{\partial \Psi}{\partial t}+\lambda \frac{\partial \Psi}{\partial \tau} \frac{d \tau}{d t}+\lambda \frac{\partial \Psi}{\partial \varepsilon} \frac{d \varepsilon}{d t}=0
$$

Because the scaling constant $\lambda \neq 0$ appears to the same power in every term of the equation, the solution is independent of the scaling.

\section{COMPARISON OF SCALED AND UNSCALED TRACKING}

In Sec. II there is a comparison of a scaled and an unscaled tracking with the collective potential generated by an $h=3 \mathrm{HOM}$ and $4 \times 10^{13}$ protons in the ring. A more realistic case is to add the space charge force to this example. In the earlier example, 8000 macroparticles were quite sufficient because the HOM resonance was at a rather low frequency. When the space charge force is evaluated, the gradient of the charge distribution is calculated; there must be enough macroparticles to produce a smooth distribution. An insufficient number results in large local fluctuation of the force and spurious breakup of the beam into small clumps. In Figs. 3 and 4 is shown the evolution of the rms energy spread of the beam as the charge is raised from zero to a final value of $2 \times 10^{13}$ protons over $0.012 \mathrm{~s}$. The unscaled example in Fig. 3 used $2 \times 10^{6}$ macroparticles and took $32 \mathrm{~h}$ of processor time on a Sun Ultra 2. The macroparticle number was selected arbitrarily and the final charge was adjusted to avoid spurious breakup. The plot in Fig. 4 was generated from tracking with the scaling $\lambda=2$ and used $2.5 \times 10^{5}$ macroparticles; it required $1.9 \mathrm{~h}$ on the same computer, just a little better than $\lambda^{4}$ 


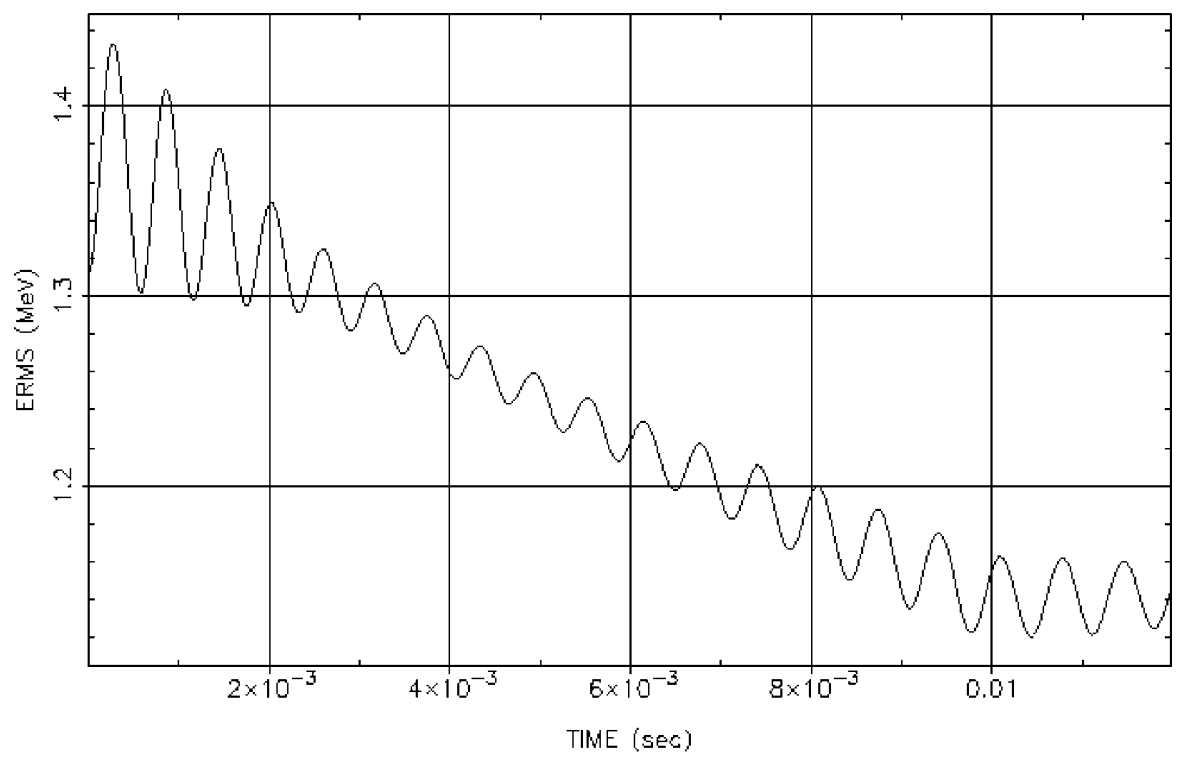

FIG. 3. The rms energy spread vs time in the ring specified in Table II for a beam intensity of $2 \times 10^{13}$ protons including both an $h=3 \mathrm{HOM}$ and the perfectly conducting wall space charge force.

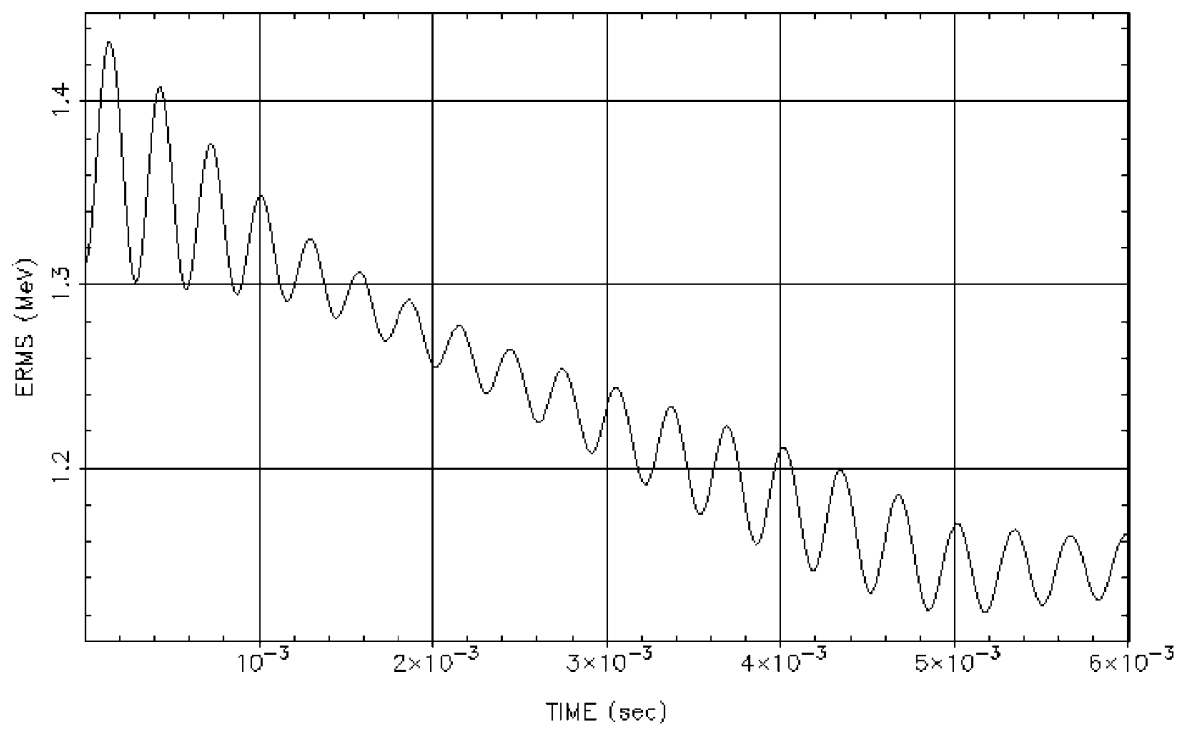

FIG. 4. The same conditions used for Fig. 3 except scaled with $\lambda=2$. Note that the time scale is $t^{\prime}=t / \lambda$, one-half of that in Fig. 3.

times faster. It may not always be possible to scale this much, although in some cases even more may be possible.

\section{UTILITY}

If the scaling factor $\lambda$ can be significantly larger than 1 without significantly reducing the accuracy of the result, much time can be saved in macroparticle modeling. One limitation on the choice of $\lambda$ has been discussed above, viz., that the spacing of harmonics of the circulation angular frequency $\omega_{0}$ should not be greater than the scale of important features of the frequency dependence of the longitudinal impedance $Z_{\|}(\omega)$. Returning to the difference Eqs. (1) and (2), one sees that, in the absence of any collective potential, the scaling by integral or $1 /$ integer $\lambda$ is equivalent to a multiturn or fractional-turn map, respectively. This indicates that an additional feature of the scaling with $\lambda>1$ is the introduction of an artificial increase in the effective time step. This artificial discretization error leads to distortion of the macroparticle trajectories that can be very apparent in extreme cases. The parameter to control is the synchrotron oscillation phase advance between energy increments. When this is below $0.01 \pi$ or so, the calculation should be at least qualitatively reasonable. 
Another condition, which is generally the same as the limitation on synchrotron oscillation phase advance per iteration, is that rf phase slip per iteration should be small with respect to a bunch length, and energy increment per iteration should be small with respect to the beam energy spread. The scaling described is so simple to implement and so innocuous in typical applications that it seems reasonable to employ it when calculation time for the unscaled case is more than a few minutes. The precaution of considering the granularity of the sampling of $Z_{\|}(\omega)$ and the adjustment of the frequency of any narrow resonances requires forethought, but the test for the appropriateness of the iteration step can easily be an automatic check in the modeling code.

The conceptual discussion above has used mostly a frequency domain description for simplicity. The two examples shown, however, are pure time domain calculations using a slightly modified version of the ESME tracking code [4]. The version called ESME2001 incorporates $\lambda$ scaling as an option; it appears compatible with all other features in both frequency and time domain. The scaling of potentials, frequency, and time are automatic, but no tests for the appropriateness of the scaling constant have been included.

\section{ACKNOWLEDGEMENTS}

The scaling described above has some similarity to that developed by Andre Gerasimov for Fokker-Planck models of stochastic beam cooling [5], and this work was encouraged by earlier success in using Gerasimov's scaling rules in beam cooling simulations. Z.N. wishes to thank those at Fermilab who arranged her visit, especially the SIST committee, for their hospitality and for their efforts to provide the opportunity for this study. This work was supported by the U.S. Department of Energy under Contract No. DE-AC02-76CH03000.

[1] J. MacLachlan, Fermilab Note No. FN-529, 1989.

[2] J. Wei, Ph.D. dissertation, State University of New York at Stony Brook, 1990.

[3] J. A. MacLachlan, FERMILAB-Conf-92/333, 1992.

[4] The current documentation, containing references to the underlying principles, is most accessible on the ESME web page http://www-ap.fnal.gov/ESME. See also, James A. MacLachlan and Jean-Francois Ostiguy, Technology Report No. FERMILAB TM-2132, 2000 (unpublished).

[5] A. Gerasimov, FERMILAB-Pub-94/376, 1994. 